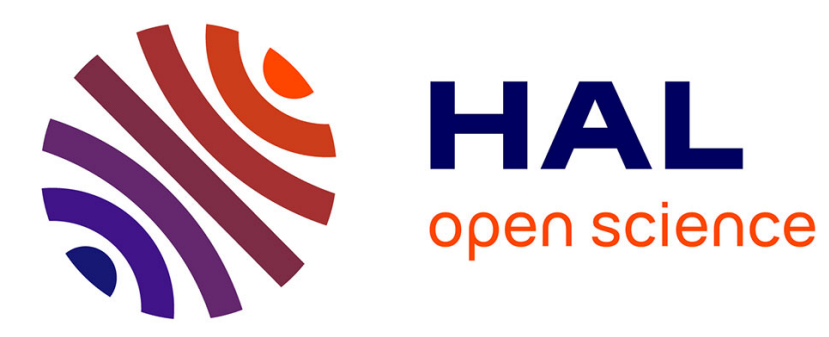

\title{
Welfare as Equity Equivalents
}

Loïc Berger, Johannes Emmerling

\section{To cite this version:}

Loïc Berger, Johannes Emmerling. Welfare as Equity Equivalents. Journal of Economic Surveys, 2020, 34 (4), pp.727-752. 10.1111/joes.12368 . hal-02937705

\section{HAL Id: hal-02937705 https://hal.science/hal-02937705}

Submitted on 18 Sep 2020

HAL is a multi-disciplinary open access archive for the deposit and dissemination of scientific research documents, whether they are published or not. The documents may come from teaching and research institutions in France or abroad, or from public or private research centers.
L'archive ouverte pluridisciplinaire HAL, est destinée au dépôt et à la diffusion de documents scientifiques de niveau recherche, publiés ou non, émanant des établissements d'enseignement et de recherche français ou étrangers, des laboratoires publics ou privés. 


\title{
Welfare as Equity Equivalents*
}

\author{
Loïc Berger ${ }^{\dagger}$ and Johannes Emmerling ${ }^{\ddagger}$
}

\begin{abstract}
Equity (or, its counterpart, inequity) plays a fundamental role in the evaluation of social welfare in different dimensions. In this paper, we revisit the concept of inequity -in the sense of unequal distributions- across individuals, time, and states of the world using a unified framework that generalizes the standard expected discounted utilitarianism approach. We propose a general measure of welfare as equity equivalents and a corresponding inequity index. We show that allowing for different attitudes toward inequity across different dimensions covers a scope of possible inequity preferences with different interpretations. We then prove that the order of aggregation across the different dimensions matters for welfare evaluations. Finally, we show that many of the welfare-theoretical approaches recently developed in the literature can be interpreted as special cases of this general framework.
\end{abstract}

Keywords: Utilitarianism, Inequality, Inequity Aversion, Risk aversion, Intertemporal Welfare, Discounting

JEL Classification: D60, D63, D30

${ }^{*}$ We thank Matthew Adler, Benoit Decerf, Marc Fleurbaey, Paolo Piacquadio, Philippe Weil and Stéphane Zuber as well as seminar participants at EAERE, IAMC, and PSE, and two anonymous referees for their useful comments. This research 1 has received funding from the Agence Nationale de la Recherche (ANR), under grant ANR-17-CE03-0008-01 (project INDUCED), from the European Research Council under the European Community's Programme "Ideas" - Call identifier: ERC-2013-StG/ERC grant agreement n³36703 - project RISICO "RISk and uncertainty in developing and Implementing Climate change pOlicies", the European Union's Horizon 2020 research and innovation programme under grant agreement $\mathrm{n}^{\circ} 776479$ - project $\mathrm{COACCH}$, from the European center for humanities and social sciences (MESHS-Lille, France), and by the French Ministry of Higher education, research and innovation. A previous version of this paper was circulated under the title "Welfare as Simple(x) Equity Equivalents".

${ }^{\dagger}$ CNRS, IESEG School of Management , Univ. Lille, UMR 9221 - LEM, F-59000 Lille, France; RFF-CMCC European Institute on Economics and the Environment (EIEE), Centro EuroMediterraneo sui Cambiamenti Climatici, and Università Bocconi, Milan, Italy. Orcid number: 0000-0002-0696-9387.

${ }^{\ddagger}$ RFF-CMCC European Institute on Economics and the Environment (EIEE), Centro EuroMediterraneo sui Cambiamenti Climatici, Via Bergognone 34, 20144 Milan, Italy. E-mail: johannes.emmerling@eiee.org. Orcid number: 0000-0003-0916-9913. 


\section{Introduction}

Distributional issues are at the heart of economics. Problems related to allocation efficiency, distribution of consumption, wealth, and other socio-economic variables have been at the core of economic analyses since the emergence of the discipline. In particular, distributional issues across individuals -be it within national boundaries (redistribution of resources) or across countries (economic development)- continue to be an important topic of research. Yet distributional issues have typically not been considered only along the individual dimension. The distribution of resources over time and the related questions of savings, intergenerational distribution stemming from capital dynamics, or the intertemporal use of natural resources have added another dimension to the problem. Finally, distributions across different "states of the world" due to the presence of uncertainty affecting the realizations of random variables have surrounded virtually all economic problems.

While the literature has historically considered the fundamentally different dimensions of individuals, time and states of the world separately, it is now clear that different potential dimensions of "inequity" (i.e. unequal distribution in a particular dimension) are potentially closely intertwined: inequality between contemporaneous individuals might be correlated with intergenerational inequity between generations, uncertainty might affect individuals differently, and so on. Focusing on one dimension of inequity in isolation therefore runs the risk of neglecting potentially important interaction effects. In this paper, we outline a unified approach for the study of inequity in different dimensions. Specifically, we consider inequity across the three dimensions of individuals (i.e. inequality issue), time (i.e. inter-generational inequity issue) and states of the world (i.e. uncertainty issue). Using a general framework that encompasses most of the approaches recently proposed in the literature on welfare and inequity, we analyze the implications of considering multiple dimensional inequity issues jointly.

Background Economics has traditionally tried to represent societies' basic objectives that guide policy-making by means of social welfare functions (SWFs). In principle, any policy recommendation regarding the allocation of resources should take these objectives into account. SWFs serve as tools for coherently organizing our thoughts about welfare and its distribution over different dimensions. To do so, they need to be defined and characterized. In particular, as SWFs may be used to advise on resource allocations, the way they are formulated may also have important policy implications. The workhorse model that has frequently been followed to aggregate 
different dimensions of social welfare is the weighted utilitarian (WU) criterion. ${ }^{1}$ In line with this approach, an SWF may be constructed by considering the utility $U_{n t s}$ of an individual $n$, at time $t$, in state of the world $s$. Social welfare then simply consists of the weighted sum of the different utilities over individuals, time, and states of the world. That is, social welfare represents the threefold (weighted) sum of utilities

$$
S W F=\sum_{n, t, s} q_{n t s} U_{n t s},
$$

where $q_{n t s}$ is a scalar representing the weight associated with utility $U_{n t s}$ evaluated in the three dimensions " $n, t, s$ ". The individual, time, and state dimensions can be considered in isolation or can be combined in different ways. In this sense, the weights $q_{n t s}$ have different interpretations depending on the dimension considered. When aggregating over individuals, they typically represent individual weights (in case they are equal, they may be omitted). In the time dimension, they usually refer to a (relative) discount factor. Finally, in the state dimension, they represent probabilities. ${ }^{2}$ The WU approach has several advantages, as it is grounded on a set of desirable axioms, defined across the three dimensions separately (see Koopmans, 1960; von Neumann and Morgenstern, 1944; and Maskin, 1978). Combining the respective one-dimensional welfare aggregations together therefore seems, at first glance, a natural extension and generalization of various one-dimensional concepts. In accordance with the discussion of Fleming (1952) on cardinal welfare in the three dimensions, the SWF approach formalized by Harsanyi (1955) presented in expression (1) has indeed been the most widely used criterion to evaluate welfare. Yet alternative approaches have also emerged.

This paper In this paper, we revisit and categorize many of the alternative social welfare concepts and specifications that have been proposed in the literature. We then discuss how standard analyses considering a single dimension of the inequity issue can be naturally extended when considering the other dimensions simultaneously. In particular, our contribution is fourfold. (i) We propose a way to articulate the different dimensions by providing a unifying welfare concept based on "equity equivalents". (ii) We then show how allowing for different attitudes toward inequity across different dimensions covers a scope of possible inequity preferences with dif-

\footnotetext{
${ }^{1}$ It is also sometimes called expected discounted utilitarian or EDU criterion in the literature.

${ }^{2}$ In what follows, we typically assume, as in Harsanyi (1955), that these probabilities are objectively known.
} 
ferent interpretations. (iii) Comparing our welfare measure with the workhorse WU criterion, we also show how the order of aggregation matters for welfare considerations. (iv) Finally, we show how most of the welfare-theoretical approaches recently developed in the literature can be interpreted as special cases of our general framework.

The paper proceeds as follows. In Section 2, we review recent literature that has investigated inequity issues in one or more dimensions. In Section 3, we introduce a unified framework generalizing the utilitarianism paradigm in one dimension and show that many of the existing welfare concepts can be deemed as special cases of this general framework. We also compute a generalized inequity index and provide numerical estimates of inequity in the three dimensions of individual, time and state separately. In Sections 4 and 5, we extend the analysis to a multi-dimensional framework that takes into account two or more dimensions simultaneously. In Section 6 , we pay particular attention to the time dimension and propose a recursive formulation of our general welfare formulation. Section 7 concludes the paper.

\section{Related literature}

A large part of the economics literature has explicitly or implicitly focused on the study of a general idea of inequity. While it is often convenient to abstract from multi-dimensional considerations and focus on one particular dimension when studying specific problems, most economic issues encompass multi-dimensional components. In particular, the discussion on the implications of climate change have spawned a wealth of literature in welfare economics and normative philosophy. Issues that have been raised in this context include inter-generational inequity (e.g. the social discount rate), the notion of inequality and distributional justice, and the role of (deep) uncertainty together with the related idea of a precautionary principle. The common feature across these seemingly unrelated concepts is that losses and benefits of given policies need to be compared along different dimensions. As the climate change problem is a particularly well-adapted example that combines the three dimensions of individuals, time and states together, in what follows, we specifically focus on (but do not limit ourselves to) reviewing approaches that have gone beyond the study of one dimensional distributions in this specific context. Importantly, we restrict the analysis to a particular class of welfare concepts of the nested additively separable class, so that welfare can be expressed, in each dimension, as a (weighted) sum of utilities. Other approaches such as dual approaches based on a 
transformation of weights and rank-dependent functions also use alternative welfare functions in each of the dimensions, but they are beyond the scope of this paper.

Time and beyond Recently, the need to aggregate welfare over long time horizons has been at the core of the discounting debate. Initially, only the time dimension of the problem was considered (Ramsey, 1928; Baumol, 1968). However, recent extensions and generalizations of the discount rate formula have also incorporated other dimensions. In particular, the discussion has been extended to take into account the state dimension, for example when incorporating the uncertainty related to economic growth (Gollier et al., 2008) or focusing on uncertain individual discount rates (Gollier and Weitzman, 2010). In the same vein, research has explored the question of discounting along the individual dimension, focusing for example on the heterogeneity of discount rates (Gollier and Zeckhauser, 2005; Feng and Ke, 2018) or considering explicitly the question of inequality (Gollier, 2015). This literature has thus primary focused on the time dimension, while introducing elements of the state and individual dimensions, respectively.

States of the world and beyond In the states of the world dimension, risk considerations have typically been realized under the expected utility (EU) framework. When an additional dimension is considered, allowing for distinct attitudes toward different dimensions, the aggregation of welfare across dimensions becomes less trivial. Notably, the approaches disentangling inter-temporal and risk preferences proposed by Selden (1978) and Kreps and Porteus (1978) and later extended by Epstein and Zin (1989) and Weil $(1989,1990)$ have been widely applied in many different fields of economics. These approaches, however, are primary oriented to the time dimension as they are geared toward preserving dynamic consistency and independence of unrealized and past alternatives, while departing from the independence axiom of the EU theory. Alternatively, a less widely used formulation, which dates back to Kihlstrom and Mirman (1974), also disentangles preferences for risk and time but focuses on maintaining the linearity in probabilities. ${ }^{3}$ In the context of climate change economics, several authors have used the Epstein-Zin/Weil framework to evaluate welfare across time and states of the world. Examples of studies using this welfare framework include Ha-Duong and Treich (2004), Crost

\footnotetext{
${ }^{3}$ From a practical standpoint, this approach has received little attention because of the importance attached to time consistency issues and to the very convenient recursive formulation proposed by the Epstein-Zin/Weil framework. A notable exception is Bommier (2007).
} 
and Traeger (2014), Ackerman et al. (2013), and Lontzek et al. (2015). ${ }^{4}$ In general, these applications rely on global analytical or numerical models that consider the world as a whole -often based on Nordhaus's (1993) DICE model ${ }^{5}$ - and thus abstract from heterogeneity across individuals. In the more welfare economic-oriented literature, extensions toward more general welfare criteria, for example considering ex-ante and ex-post concepts of prioritarianism, include Adler and Treich (2017). ${ }^{6}$

Individuals and beyond The third dimension traditionally analyzed in the literature concerns inequality across individuals. ${ }^{7}$ When considering distributional issues in the individual dimension in a global or international context, as in the context of climate change, individuals typically refer to countries (or group of countries). In most optimization models with country or regional disaggregation, the issues of inequality and distributional effects have typically been left aside by using Negishi weights (Nordhaus and Yang, 1996). This approach however has several conceptual flaws (Stanton, 2011), which could make alternative welfare approaches more desirable. In particular, employing the measurement of inequality pioneered by Pigou (1912); Dalton (1920); and Atkinson (1970) several studies have recently applied similar concepts to the modeling of global policy issues. Note that there is also a wealth of literature that has extended this work to capture multi-dimensional inequality issues, such as inequality across individuals (or countries), but along different dimensions such as income, health, and education (Maasoumi, 1986; Tsui, 1995; Decancq et al., 2009).

In the context of climate change, the discussion on "equity weights" has, since Fankhauser et al. (1997), received a great deal of attention. The general idea relies on extending standard welfare measures over time to include inequality considerations

\footnotetext{
${ }^{4}$ See also Berger et al. (2017) for an application using an extension of the Epstein-Zin/Weil formulation that goes beyond the analysis of risk, by considering deep uncertainty.

${ }^{5}$ DICE stands for Dynamic Integrated Climate and Economy, see Nordhaus (1993) and Nordhaus and Sztorc (2013) for more details.

${ }^{6}$ Prioritarianism is a welfare concept that goes beyond utilitarianism in that the welfare of worse-off individuals are given a higher weight (Adler and Fleurbaey, 2016). In our framework, using a single utility function in the one-dimensional case, it can be interpreted as a concave transformation of the utility function. In the specific case of the two dimensions time and individuals, prioritarianism has been reinterpreted to consider intertemporal welfare and the distribution within generations across individuals (Adler, 2012; Adler and Treich, 2017). These welfare frameworks can be comprised in the general framework of this paper. Other welfare concepts include egalitarianism (an even more extreme welfare concept in which equality across individuals is prioritized), rankbased welfare concepts (Zuber and Asheim, 2012), inter-generational egalitarianism (Piacquadio, 2014), and the Rawlsian maxmin welfare function. As these are based on more complex welfare functions going beyond the paradigm of weighted utilitarianism, we do not consider them herein.

${ }^{7}$ This type of inequity is also called "intra-generational inequity".
} 
(in the spirit of Atkinson's approach) at any point in time. Since then, research has proposed a separation between the inequality concerns from time preferences (Anthoff and Tol, 2009; Tol, 2010). These studies explicitly separate inequality aversion between countries from inter-temporal fluctuations aversion and introduce the concept of a "certainty, equity, and balanced growth equivalent" level of consumption as a welfare measure. Finally, in welfare economics, the separation between individual and state dimensions has been formally analyzed by Grant et al. (2012), who build on the approaches of Fleurbaey (2010) ("expected equally distributed equivalent utility") Epstein and Segal (1992) and Diamond et al. (1967) ("generalized utilitarianism"), or by Fleurbaey and Zuber (2013).

Finally, while most of the literature has focused on one or two dimensions of the inequity issue, a few recent contributions have also proposed a combination of the three dimensions at the same time. For example, Schmidt et al. (2012) extend the intertemporal DICE model to take into account both the uncertainty and the heterogeneity across populations using a simple parametric distribution of income at any point in time. However, they do not make explicit the separation between the different attitudes over these dimensions. Fleurbaey and Zuber (2015) also consider all three dimensions in the context of the optimal discount rate. In Appendix A, we provide a graphical representation of how the existing studies on inequity relate to each dimension in a three-dimensional simplex.

\section{A unified framework for the study of inequity: the one-dimensional case}

In this section, we focus on evaluating the distribution of one variable (e.g., consumption income, health) across one dimension. We remain in the WU paradigm by assuming that the social evaluation function belongs to the nested additively separable class. In other words, we assume that welfare can be expressed as a (weighted) sum of utilities. We begin by defining a general framework for the analysis of welfare and inequity.

\subsection{General setting}

Let $C$ be a variable of interest distributed in a three-dimensional state space $\mathcal{C} \subseteq\left(\mathbb{R}^{+}\right)^{3}$. Let $G$ be a measure of $C$ over $\mathcal{C}$. We denote by $\left\{c_{n t s} \in \mathbb{R}^{+}, n \in N, t \in T, s \in S\right\}$ 
the realization of $C=\left(C_{n t s}\right)_{n \in N, t \in T, s \in S}$, which represents the consumption of individual $n$ at time $t$ in state of the word $s .{ }^{8}$ In this expression, $N, T$ and $S$ are index sets representing the individual, time, and state dimensions, respectively. These sets may be countable or uncountable. For example, the time set $T$ may represent either the (possibly infinite) discrete periods of time or continuous time. If one dimension is not considered, its respective index sets is singleton, and we simply omit it in our notation. In this sense, $c_{i}$ denotes the realized consumption level in dimension $i \in\{n, t, s\}$ only, and $c_{i j}$ is the realized consumption level in dimensions $i, j$ $\in\{n, t, s\}$ with $i \neq j$. For the sake of simplicity, we assume that the consumption (or income, health, etc.) levels are bounded. Finally, in a slight abuse of notation, we let $n, t$ and $s$ also denote the dimensional space they index when used as a superscript.

\subsection{Welfare as equity equivalent}

We now introduce a general framework that we use to analyze welfare and inequity across different dimensions. To do so, we begin by defining the concept of equity equivalent in one dimension.

Definition 1. For each $C$ of $\mathcal{C}$ and each $i \in\{n, t, s\}$, the equity equivalent of $C$ in dimension $i$, denoted by $\mathcal{E}^{i}(C)$, is the level of consumption that if assigned uniformly across dimension $i$ leaves social welfare unchanged. We formally define it as:

$$
\mathcal{E}^{i}(C) \equiv f_{i}^{-1} \mathbb{E}^{i}\left[f_{i}(C)\right]
$$

where $f_{i}: \mathbb{R}^{+} \rightarrow \mathbb{R}$ is a strictly increasing function capturing preferences over $C$ in dimension $i$ and $\mathbb{E}^{i}$ represents the expectation operator taken over dimension $i$.

Aversion toward inequity in dimension $i$ is modeled by a concave $f_{i}$, which can be interpreted as aversion to (mean preserving) spreads in consumption levels. The expectation -or weighted sum- operator takes the form $\mathbb{E}^{i}\left[f_{i}(C)\right]=\sum_{i} q_{i} f_{i}\left(c_{i}\right)$, where $q_{i}$ represents the weights attached to elements $c_{i}$ such that $\sum_{i} q_{i}=1 .{ }^{9}$ In this sense, the measure $G$ can be taken as a probability measure. We then define the one-dimensional social welfare evaluation function to rank unequal distributions according to this equity equivalent.

\footnotetext{
${ }^{8}$ The order in which indices are specified does not matter here, such that $c_{n t s}=c_{s t n}=c_{t n s}=\ldots$

${ }^{9}$ In the limiting case in which the distribution of $C$ is continuous over dimension $i$, the operator $\mathbb{E}^{i}$ is simply defined as the (Lebesgue) integral $\mathbb{E}^{i}\left[f_{i}(C)\right]=\int f_{i}(C) d G_{i}$ with respect to the marginal distribution $G_{i}$.
} 
Definition 2. The SWF over dimension $i$ is the function $W^{i}: \mathcal{C} \rightarrow \mathbb{R}^{+}$defined as

$$
W^{i}(C) \equiv \mathcal{E}^{i}(C) \quad \forall i \in\{n, t, s\}
$$

To understand why $W^{i}(C)$ can be interpreted as an index of social welfare, note that for any $C, C^{\prime} \in \mathcal{C}, \mathcal{E}^{i}(C) \geq \mathcal{E}^{i}\left(C^{\prime}\right)$ means that the distribution $C$ of consumption levels across dimension $i$ is deemed socially at least as good as the distribution $C^{\prime}$. This formulation of welfare is slightly different from the formulation $V^{i}(C)=$ $\sum_{i} q_{i}^{\prime} f_{i}\left(c_{i}\right)$-with the sum $\sum_{i} q_{i}^{\prime}$ that does not necessarily sum up to 1 - which is typically used in the literature. However, because both the re-scaling (consisting of writing $q_{i} \equiv \frac{q_{i}^{\prime}}{\sum_{i^{\prime}}^{\prime} q_{i^{\prime}}^{\prime}}$ ) and the transformation $f_{i}^{-1}$ induced by the equity equivalent formulation are strictly monotonic transformations of $V^{i}(C)$, they do not alter the ordinal properties of the welfare ranking. The certainty equivalent formulation (3) also allows for both a more intuitive interpretation of the welfare concept and easier generalizations. ${ }^{10}$ Under extreme inequity aversion, the equity equivalent (2), and thus the SWF $W^{i}$, reduces to the maxmin criterion given by ${ }^{11}$

$$
\mathcal{E}^{i}(C)=\min _{i}\left\{c_{i}\right\}
$$

\subsection{Inequity measure}

One useful characteristic of this general welfare concept is that it can be related to a general measure of inequity. In particular, we can define a general measure of inequity as follows:

Definition 3. For each $C$ of $\mathcal{C}$ and each $i \in\{n, t, s\}$, the inequity index of $C$ in dimension $i$ is defined as

$$
I^{i} \equiv 1-\frac{\mathcal{E}^{i}(C)}{\mathbb{E}^{i}[C]},
$$

where $\mathbb{E}^{i}[C] \equiv \sum_{i} q_{i} c_{i}$ is the mean of the actual distribution of $C$ across the dimension considered. ${ }^{12}$ Importantly, this inequity index depends on the underlying function $f_{i}$ used to compute the equity equivalent. If the distribution of consumption

\footnotetext{
${ }^{10}$ Another advantage of the equity equivalent formulation is that it leads to numerical values of welfare that are closer to the variable of interest in terms of orders of magnitude. This might be useful in numerical applications because it can help improve numerical optimization algorithms.

${ }^{11}$ See the discussion in Section 3.5. For a general proof, see Klibanoff et al. (2005, p. 1867).

${ }^{12} \mathbb{E}^{i}[C]$ can equivalently be obtained from (2) in the special case of neutrality toward inequity in dimension $i$ (i.e. if $f_{i}$ is an affine function).
} 
becomes more equally distributed in dimension $i$, the equity equivalent of someone who is averse to inequity rises, and the inequity index falls. The inequity index $I^{i}$ also has the convenient property of being bounded between 0 (perfect equity) and 1 (perfect inequity).

\subsection{Applications in the literature}

Different strands in the literature have proposed one-dimensional inequity measures. In what follows, we show how our general framework can capture the various existing concepts. We also discuss a new interpretation along the time dimension.

Individual dimension When considering the dimension across individuals $(i=$ $n$ ), the equity equivalent $\mathcal{E}^{n}(C)$ corresponds to the equally distributed equivalent (EDE, see Atkinson, 1970; Fleurbaey, 2010). The EDE represents the level of consumption that, if it were equally distributed over individuals, would give the same level of welfare as the actual distribution of consumption. In this context, $g \equiv f_{n} \forall n$ is a function capturing preferences toward inequality across individuals and $q_{n}$ corresponds to the relative weight attached to individual $n$. In the same vein, $I^{n}$ corresponds to Atkinson's (1970) inequality index.

State dimension For the state dimension $(i=s)$, the equity equivalent $\mathcal{E}^{s}(C)$ corresponds to the standard certainty equivalent $(\mathrm{CE})$ defined over the uncertain variable $C$ in the risk theory literature. In this context, $s$ represents the different states of the world; $v \equiv f_{s} \forall s$ is the von Neumann-Morgenstern cardinal utility capturing preferences toward risk; and $\mathbb{E}^{s}$ is the expectation operator with $q_{s}$ representing the probability of being in state $s$, in which the level of consumption is $c_{s}$. Finally, note that $\mathbb{E}^{s}[C]$ represents the expected value of $C$ and $I^{s}$ is the relative risk premium. ${ }^{13}$

Time dimension For the time dimension $(i=t)$, the equity equivalent $\mathcal{E}^{t}(C)$ represents the variable level, constant over time, that would give the same social welfare as the actual stream of $\left(C_{t}\right)_{t \in T}$. In this case, the function $u \equiv f_{t} \quad \forall t$ captures the attitude toward intertemporal fluctuations (i.e. preferences toward unequal distribution of consumption over time). Note that this attitude toward intertemporal

\footnotetext{
${ }^{13}$ The relative risk premium corresponds to the share of the mean consumption that one is ready to pay to get rid of a risk on the consumption level. It is implicitly defined by $\sum_{s} q_{s} u\left(c_{s}\right)=$ $u\left(\mathbb{E}^{s}[C]\left(1-I^{s}\right)\right.$ ), (see Eeckhoudt et al., 2005). Notably, it has the advantage to be a unit-free measure contrary to the absolute risk premium.
} 
fluctuations may pertains to either the same individual living at different periods of time or the same dynasty (or family) made of different generations linked through operative inter-generational transfers. In the latter case, $f_{t}$ could be interpreted as capturing inequity preferences across generations. The relative weights, $q_{t}$, represent utility discounting, potentially coupled with changes of generation size over time. ${ }^{14}$

This concept of equity equivalent over the time dimension is closely related to the notion of balanced growth equivalent (BGE) introduced by Mirrlees and Stern (1972). ${ }^{15}$ In particular, the two concepts exactly coincide with a zero growth rate $(\lambda=0)$ of the BGE: $B G E_{\lambda=0}(C)=\mathcal{E}^{t}(C)$. To our knowledge, an analogous measure of the inequity index has never been defined along the time dimension. However, we can compute it easily from equation (5). This "intertemporal inequity index", $I^{t}$, provides an index of intertemporal inequity from the social planner's perspective. When $C$ represents consumption, it can be interpreted as the share of average discounted consumption that is lost from the unequal distribution over time.

Extension to other dimensions While the three dimensions we discuss are those that have been the most widely studied, the general approach we propose can be easily extended to other dimensions. For example, the set of dimensions can be extended to incorporate an element $m$ representing an extra dimension of uncertainty known as model uncertainty (Marinacci, 2015). In this case, $f_{m}$ would capture attitudes toward model uncertainty, and $q_{m}$ would represent the prior belief associated with each potential probability model. Other possible extensions include differentiated preferences over one's lifetime, over future generations, or over individuals within and between countries. Another important related topic is the literature on multi-dimensional inequality across individuals, which does not focus solely on monetary consumptions levels but considers multiple attributes, e.g., health, food consumption, environment, education (see Maasoumi, 1986; Tsui, 1995; Bosmans et al., 2015). ${ }^{16}$ However, treating these dimensions departs from our framework that focuses on variables of comparable scale belonging to the same monetary consump-

\footnotetext{
${ }^{14}$ These weights can be computed as $q_{t}=\frac{P_{t} \beta^{t}}{\sum_{t^{\prime}} P_{t^{\prime}} \beta^{t^{\prime}}}$, where $\beta$ is the utility discount factor and $P_{t}$ is the size of the generation living at time $t$.

${ }^{15}$ The BGE measures the level of today's consumption that, assuming it grows at a constant growth rate $\lambda$, would yield the same level of consumption as a given consumption path.

${ }^{16}$ Notably, aggregating across the dimensions of the Human Development Index (HDI), consumption, health, and education, Foster et al. (2005) develop a welfare-based axiomatic framework, showing that using the same welfare function to aggregate across individuals and dimensions can be desirable.
} 
tion domain.

\subsection{Constant inequity aversion}

The equity equivalent $\mathcal{E}^{i}$ and inequity index $I^{i}$ thus refer to different concepts, depending on the dimension considered. For each dimension, a particular function $f_{i}$ serves to capture inequity preferences. The concavity of $f_{i}$ may in turn capture inequality aversion, intertemporal inequity aversion, or risk aversion. In the spirit of Arrow (1964) and Pratt (1964), we can define $\alpha^{i}\left(c_{i}\right)=\frac{-f_{i}^{\prime \prime}\left(c_{i}\right)}{f_{i}^{\prime}\left(c_{i}\right)}$ and $\varphi^{i}\left(c_{i}\right)=\frac{-c_{i} f_{i}^{\prime \prime}\left(c_{i}\right)}{f_{i}^{\prime}\left(c_{i}\right)}$ as local measures of, respectively, the "absolute inequity aversion" and the "relative inequity aversion" in dimension $i$. In particular, the absolute inequity aversion is constant and equal to $\alpha^{i}>0$ if the $f_{i}$ function is of the exponential type

$$
f_{i}(x)=-\frac{1}{\alpha^{i}} e^{-\alpha^{i} x}
$$

Alternatively, the relative inequity aversion is constant and equal to $\varphi_{i}>0$ if the $f_{i}$ function is of the isoelastic type

$$
f_{i}(x)= \begin{cases}\frac{x^{1-\varphi^{i}}}{1-\varphi^{i}} & \text { if } \varphi^{i} \neq 1 \\ \ln (x) & \text { if } \varphi^{i}=1\end{cases}
$$

The constant absolute inequity aversion formulation (6) is particularly useful to illustrate our limit result provided in expression (4). In this case, the equity equivalent is written as

$$
\mathcal{E}_{\alpha^{i}}^{i}(C)=-\frac{1}{\alpha^{i}} \ln \sum_{i} q_{i} e^{-\alpha^{i} c_{i}},
$$

where $\alpha^{i}>0$. Here, when inequity aversion goes to infinity $\left(\alpha^{i} \rightarrow \infty\right)$, we can easily show a return to the maxmin criterion:

$$
\lim _{\alpha^{i} \rightarrow \infty} \mathcal{E}_{\alpha^{i}}^{i}(C)=\min _{i}\left\{c_{i}\right\} .
$$

The literature has also widely used the isoelastic formulation (7) in various dimensions. In particular, research has used it in the context of the Ramsey rule for social discounting, in the context of inequality for computing the family of Atkinson's indices of inequality measurement, and in the risk theory literature as a function exhibiting constant relative risk aversion (CRRA). For this special case, and to make the dependence of the inequity index $I^{i}$ on the degree of inequity aversion evident, we denote by $I^{i}\left(\varphi^{i}\right)$ the degree of inequity in the special case of isoelastic function 
$f_{i}$ with parameter $\varphi^{i}$.

In Online Appendix S1, we discuss and compare the different degrees of inequity aversion that have been typically used in the literature. In Online Appendix S2, we then use actual data to further illustrate the comparability of our general inequity index across different dimensions.

\section{A unified framework for the study of inequity: the two-dimensional case}

As argued previously, there may be many situations in which different dimensions simultaneously affect the distribution of the variable of interest. As a consequence, welfare considerations and inequity issues are generally not analyzed in uni-dimensional contexts, but rather among several dimensions simultaneously. In this section, we extend the scope of our framework to analyze inequity issues by allowing for different attitudes toward two dimensions of inequity. We then show how our framework covers different welfare criteria recently proposed in the literature.

\subsection{Two-dimensional equity equivalent and inequity mea- sure}

When we move from a one-dimensional distribution to a multi-dimensional one, different orders of aggregation become possible. ${ }^{17}$ We begin by defining the notions of welfare and inequity in terms of equity equivalents, before comparing these different orders of aggregation.

Definition 4. The SWF over the ordered dimensions $i$ and $j$ is the function $W^{i j}$ : $\mathcal{C} \rightarrow \mathbb{R}^{+}$defined as

$$
W^{i j}(C) \equiv \mathcal{E}^{i}\left(\mathcal{E}^{j}(C)\right) \quad \forall i, j \in\{n, t, s\} \text { with } i \neq j,
$$

\footnotetext{
${ }^{17}$ Using different sets of axioms, Tsui (1995) and Gajdos and Weymark (2005) discussed the role of different orders of aggregation when evaluating multi-dimensional inequality measures. In particular, in this literature, multi-dimensional Atkinson-based measures (Weymark, 2006; Aristei and Bracalente, 2011) and generalized Gini indices (Weymark, 2006; Decancq and Lugo, 2012) have been developed. Yet the "dimensions" in that literature all refer to the same qualitative dimension (attributes of individuals), whereas we focus on the different dimensions of individuals, time, and states of the world. A notable exception is Gajdos and Maurin (2004), who provide an axiomatic characterization of social welfare functions under under uncertainty.
} 
where $W^{i j}(C)$ captures the preferences of a social planner who first aggregates consumption through dimension $j$ by considering the equity equivalent $\mathcal{E}^{j}$, before aggregating it through dimension $i$, using $\mathcal{E}^{i}$. In the special case in which the two functions $f_{i}$ and $f_{j}$ capturing the social planner's attitudes toward dimensions $i$ and $j$ are identical, this welfare measure collapses to the standard WU equivalent defined as

$$
W^{\mathrm{WU}}(C) \equiv f^{-1}\left(\mathbb{E}^{i} \mathbb{E}^{j}[f(C)]\right) \quad \forall i, j \in\{n, t, s\} \text { with } i \neq j .
$$

In this special case, the order of aggregation does not matter, and the function $f=f_{i}=f_{j}$ captures attitudes toward inequity in both dimensions $i$ and $j$.

Definition 5. Analogously to expression (5), we define the two-dimensional inequity index of $C$ as

$$
I^{i j} \equiv 1-\frac{\mathcal{E}^{i}\left(\mathcal{E}^{j}(C)\right)}{\mathbb{E}^{i} \mathbb{E}^{j}[C]},
$$

where $\mathbb{E}^{i} \mathbb{E}^{j}[C]$ represents the mean of $C$ taken over both dimensions $i$ and $j$.

For the welfare measure, it should be clear that the order of aggregation in the case of identical inequity preferences for dimensions $i$ and $j$ does not affect this index. We formally discuss this result in the following paragraphs.

\subsection{Welfare and inequity comparisons for different orderings}

In this section, we formally compare different orders of aggregation. The first proposition and its corollary summarize what was previously observed when attitudes toward inequity are the same in each dimension. Their proofs trivially follow from the separability across dimensions of the aggregation (equity equivalent) operator.

Proposition 1. Let $f_{i}$ and $f_{j}$ be two functions capturing inequity attitudes in dimensions $i$ and $j$, respectively. If $f_{i}$ and $f_{j}$ are cardinally equivalent (i.e. if $f_{i}=a f_{j}+b$ where $a, b \in \mathbb{R}$ and $a \neq 0$ ), the order of aggregation does not matter, and the social welfare measure is the standard $W U$ equivalent (9).

Conversely, when the attitudes of the social planner toward different dimensions are distinct, the order of aggregation does affect the level of welfare and its corresponding measure of inequity. We now derive conditions on how the levels of welfare can be compared for the different orders of aggregation. We first introduce a useful 
lemma before stating a general result that holds for any pair of combinations of the dimensions $n, t$ and $s$.

Lemma 1. Let $f_{i}$ be a four times continuously differentiable, strictly increasing and strictly concave (convex) function. Then, the equity equivalent $\mathcal{E}^{i}(C)$ is concave (convex) if and only if the absolute inequity tolerance $-f_{i}^{\prime} / f_{i}^{\prime \prime}$ is concave (convex).

Proof. All proofs are relegated to Appendix B.

This lemma enables us to compare the two welfare functions $W^{i j}(C)$ and $W^{j i}(C)$ in the trivial case in which the attitude toward one of the two dimensions is neutral. For example, if $f_{j}$ is linear, Lemma 1 indicates that $W^{i j}(C) \geq W^{j i}(C)$, provided that the absolute inequity tolerance of $f_{i}$ is concave. ${ }^{18}$ When both attitudes toward inequity are non-neutral, extra conditions emerge, as summarized in the following proposition:

Proposition 2. Let $f_{i}$ and $f_{j}$ be strictly increasing, strictly concave, four times continuously differentiable functions. Suppose that $\phi \equiv f_{i} \circ f_{j}^{-1}$ exhibits concave absolute inequity tolerance. Then, it holds that $W^{i j}(C) \geq W^{j i}(C)$ if and only if $f_{i}$ is strictly more concave than $f_{j}$ (i.e. $\left.-f_{i}^{\prime \prime} / f_{i}^{\prime}>-f_{j}^{\prime \prime} / f_{j}^{\prime}\right)$.

This result shows that it is possible to compare different orders of aggregation in terms of welfare in the general case, under a set of conditions both on the relative concavity of the functions $f_{i}$ and $f_{j}$, and on the concavity of a composition of them. The interpretation of this latter condition is not trivial though.

To gain further intuition, we now focus on the special case in which functions $f_{i}$ and $f_{j}$ are of the isoelastic type. In Appendix $\mathrm{C}$, we also treat the more general class of harmonic absolute inequity aversion functions (also called harmonic absolute risk aversion [HARA])).

Proposition 3. Let $f_{i}$ and $f_{j}$ be of the isoelastic type, with coefficients of relative inequity aversion $\varphi_{i}>0$ and $\varphi_{j}>0$, respectively. Then,

(i) $W^{i j}(C)>W^{j i}(C)$ if and only if $\varphi^{i}>\varphi^{j} \neq 1$, unless $\exists a \in \mathbb{R}$ s.t. $c_{i^{\prime} j}=a c_{i j}$ $\forall i, j \in\{n, t, s\}$ with $i \neq j$;

(ii) $W^{i j}(C)>W^{j i}(C)$ if and only if $\varphi^{i}>\varphi^{j}=1$, unless $\exists b \in \mathbb{R}$ s.t. $c_{i^{\prime} j}=c_{i j}+b$ $\forall i, j \in\{n, t, s\}$ with $i \neq j$;

\footnotetext{
${ }^{18}$ As expressed in Lemma 1, the absolute inequity tolerance refers to the inverse of its absolute inequity aversion (see Gollier, 2001). Note that this tolerance is concave if $f_{i}$ belongs to the class of functions exhibiting harmonic absolute inequity aversion (see Appendix $\mathrm{C}$ for more details). This class of functions includes the isoelastic, exponential, and quadratic functions as special cases.
} 
(iii) $W^{i j}(C)=W^{j i}(C)$ if and only if either $\varphi^{i}=\varphi^{j} \neq 1$ or $\exists a \in \mathbb{R}$ s.t. $c_{i^{\prime} j}=a c_{i j}$ $\forall i, j \in\{n, t, s\}$ with $i \neq j$; and

(iv) $W^{i j}(C)=W^{j i}(C)$ if and only if either $\varphi^{i}=\varphi^{j}=1$ or $\exists b \in \mathbb{R}$ s.t. $c_{i^{\prime} j}=c_{i j}+b$ $\forall i, j \in\{n, t, s\}$ with $i \neq j$.

The intuition behind these results is simple. First, the two cases must be separated depending on the degree of inequity aversion in dimension $j$. The first case $\left(\varphi^{j} \neq 1\right)$ implies that the combination $\phi \equiv f_{i} \circ f_{j}^{-1}$ is itself of the isoelastic type, while the second $\left(\varphi^{j}=1\right)$ implies that $\phi$ is of the exponential type. Then, Proposition 3 indicates that welfare will be higher when aggregating first over the dimension that is associated with the lower level of inequity aversion, unless the consumption stream is either multiplicative or additive (depending on the functional form of $\phi$ ) in dimension $i$, in which case the order of aggregation does not matter. ${ }^{19}$

Finally, another important difference between the possible welfare orderings is the correlation structure of $C$ (see the following example and the discussion in Bommier, 2007). While clear-cut results are difficult to obtain in general, in the case of independence between the dimensions, it is possible to show the following:

Proposition 4. If $C$ is independently distributed across dimensions, that is, $G(C)=$ $G_{i}(C) G_{j}(C)$, the order of aggregation does not matter for welfare evaluation.

In this case indeed, $W^{i j}(C)=W^{j i}(C)$, and the next result trivially follows.

Corollary 1. Under the assumption of Proposition 4, we can compute the twodimensional inequity index as $I^{i j}=I^{i}+I^{j}-I^{i} I^{j}$.

\subsection{Applications in the literature}

With this set-up in mind, we now consider some particular cases of our general twodimensional framework. In particular, we connect them with the various welfare criteria that have been proposed in the literature. ${ }^{20}$

\footnotetext{
${ }^{19} \mathrm{~A}$ consumption stream that is multiplicative in dimension $i$ such that $\exists a \in \mathbb{R}$ s.t. $c_{i^{\prime} j}=a c_{i j}$ $\forall i, j$, is automatically also multiplicative in dimension $j: \exists a \in \mathbb{R}$ s.t. $c_{i^{\prime} j}=a c_{i j} \forall i, j \Longleftrightarrow \exists a^{\prime} \in$ $\mathbb{R}$ s.t. $c_{i j^{\prime}}=a^{\prime} c_{i j} \forall i, j$. The same property holds for the additive case.

${ }^{20} \mathrm{As}$ previously, we use $g, u$, and $v$ to represent the functions capturing attitudes toward the individual, time, and state dimensions, respectively.
} 
Time and state If the two dimensions considered simultaneously are time and state and a single-agent is considered, the equity equivalent formulation (8) is equivalent to the well-known criteria proposed by Selden (1978); Kreps and Porteus (1978), or Kihlstrom and Mirman (1974). These criteria have been widely used and applied in many different contexts in economics. To show the link with our formulation, we consider the case with two periods (i.e. $|T|=2$ ) and let $C_{t}$ denote $\left(C_{t s}\right)_{s \in S}$. We also assume that first-period consumption, $C_{1}$, is deterministic and that second-period consumption, $C_{2}$, is risky. When the aggregation is first realized over states of the world and then over time, the equity equivalent (8) is

$$
W^{t s}(C)=u^{-1}\left(\frac{1}{1+\beta} u\left(C_{1}\right)+\frac{\beta}{1+\beta} u \circ v^{-1}\left(\mathbb{E}^{s}\left[v\left(C_{2}\right)\right]\right)\right) .
$$

Similarly, if the order of aggregation is first realized over time and then over states of the world, the equity equivalent (8) becomes

$$
W^{s t}(C)=v^{-1}\left(\mathbb{E}^{s}\left[v \circ u^{-1}\left(\frac{1}{1+\beta} u\left(C_{1}\right)+\frac{\beta}{1+\beta} u\left(C_{2}\right)\right)\right]\right) .
$$

When $u=v$, both expressions $W^{t s}(C)$ and $W^{s t}(C)$ collapse to the equity equivalent version of the standard expected value of discounted utility. Using this framework, we can easily show that criterion $W^{t s}(C)$ in expression (11) is just a monotonic transformation of the intertemporal welfare criterion proposed by Selden $(1978,1979)$ and Kreps and Porteus (1978) (S-KP) and is usually written as

$$
W^{S-K P}(C)=u\left(C_{1}\right)+\beta u \circ v^{-1}\left(\mathbb{E}^{s}\left[v\left(C_{2}\right)\right]\right) .
$$

Analogously, criterion $W^{s t}(C)$ in expression (12) corresponds to the equity equivalent version of Kihlstrom and Mirman's (KM, 1974) criterion in the context of a twoperiod, additively separable model (see Bommier et al., 2012). To see this, note that the KM criterion is usually written as

$$
W^{K M}(C)=\mathbb{E}^{s}\left[\varphi\left(u\left(C_{1}\right)+\beta u\left(C_{2}\right)\right)\right],
$$

where $\varphi$ is increasing and concave and $\varphi \equiv v \circ u^{-1}$. However, the concavity of $\varphi$ amounts to $v$ being more concave than $u .{ }^{21}$ Finally, when preferences are represented

\footnotetext{
${ }^{21} v$ more concave than $u$ implies the existence of a strictly increasing and concave function $k$, such that $v=k \circ u$.
} 
by functions of the isoelastic type, the formulation $W^{t s}(C)$ is also equivalent to the widely used Epstein and Zin (1989) and Weil (1989, 1990) (EZW) preferences (see Section 6 for cases when there are more than two periods), which can be written as

$$
W^{E Z W}(C)=\frac{C_{1}^{1-\eta}}{1-\eta}+\beta \frac{1}{1-\eta}\left(\mathbb{E}^{s}\left[\left(C_{2}^{1-\rho}\right]\right)^{\frac{1-\eta}{1-\rho}},\right.
$$

where $1 / \eta$ is the intertemporal elasticity of substitution and $\rho$ is the coefficient of relative risk aversion. With such isoelastic functions, we know from Proposition 3 that if relative risk aversion is stronger than the inverse of the intertemporal elasticity of substitution, the welfare evaluated under Selden's (1978) preferences is generally higher than that under Kihlstrom and Mirman's (1974) preferences.

Alternatively, if the problem is one of inter-generational equity between different generations of individuals (i.e. multi-agent problem), our general formulation may also be encompassing different SWFs that have been proposed in the literature. The first and most obvious one is the utilitarian (U) criterion, which is recovered by letting $\beta=1$ (i.e. future generations' utility is not discounted) and $u=v$. In this case, our $W^{W U}(C)$ is equivalent to

$$
W^{U}(C)=v\left(C_{1}\right)+\mathbb{E}^{s}\left[v\left(C_{2}\right)\right]
$$

Under this criterion, the two generations have the same utility function $v$, but the risk is only born by the second generation. Alternative welfare criteria that have been proposed instead follow the prioritarian approach, by associating greater weight with changes in well-being affecting worse-off generations. These include ex ante prioritarianism (EAP) and ex post prioritarianism (EPP) (see Adler and Treich, 2017). The main difference between utilitarianism and prioritarianism is that the latter takes into account total well-being and the distribution of well-being over generations. Under EAP, the SWF is written as

$$
W^{E A P}(C)=(\phi \circ v)\left(C_{1}\right)+\phi\left(\mathbb{E}^{s}\left[v\left(C_{2}\right)\right]\right),
$$

where $\phi$ is an increasing, concave function transforming generations' expected utilities. In this case, the SWF consists of a sum of transformed expected utilities, in accordance with the ex ante approach. By denoting $\phi \equiv u \circ v^{-1}$, we can then easily show that the notion of $W^{E A P}(C)$ and our formulation $W^{t s}(C)$ are equivalent (recall that $\beta=1$ ). In this case, the concavity of $\phi$ amounts to $u$ being more concave than 
$v$. Under EPP, the SWF is written as

$$
W^{E P P}(C)=(\phi \circ v)\left(C_{1}\right)+\mathbb{E}^{s}\left[(\phi \circ v)\left(C_{2}\right)\right] .
$$

In this case, welfare comprises the expectation of transformed utilities, in line with the ex post approach. The concavity of $\phi$ captures inequity aversion in the space of utility or welfare and not in the space of consumption levels. Under concavity of $\phi$, this criterion takes the same form as (16) (and thus is equivalent to $W^{W U}(C)$ ) but this time is evaluated under a more concave function (capturing stronger aversion toward inequity).

State and individual If, instead, we consider the atemporal distribution of risks over individuals, we can relate our general formulation to recent welfare criteria that have been proposed to assess social situations involving risk. In particular, we can write the two welfare functions $W^{s n}(C)$ and $W^{n s}(C)$ as

$$
W^{n s}(C)=g^{-1}\left(\sum_{n \in N} q_{n} g \circ v^{-1}\left(\sum_{s \in S} q_{s}\left[v\left(c_{n s}\right)\right]\right)\right)
$$

and

$$
W^{s n}(C)=v^{-1}\left(\sum_{\mathrm{s} \in \mathrm{S}} q_{s}\left[v \circ g^{-1}\left(\sum_{n \in N} q_{n} g\left(c_{s n}\right)\right)\right]\right),
$$

where $c_{n s}=c_{s n}$ is the realized level of consumption for individual $n$ in state of the world $s$. In the special case when $v=g$, the two SWFs collapse to

$$
W(C)=v^{-1}\left(\sum_{n \in N} q_{n} \sum_{s \in S} q_{s} v\left(c_{n s}\right)\right)
$$

Criterion (21) is the equity equivalent version of the standard utilitarian approach in which social welfare is the sum of individual expected utilities or equivalently the expected value of the sum of individual utilities (Harsanyi, 1955). In welfare economics, the individual weights are typically assumed to be uniform $q_{n}=1 /|N|$. Yet, as we already noted when dealing with different generations, a disturbing feature of utilitarianism is its indifference to the distributions of utilities. As is now clear, the absence of preference for equality in utility terms comes from the equal treatment of state and individual (i.e. $v=g$ ). Research has proposed different alternative approaches that accommodate such shortcoming. For example, Diamond et al. (1967) and Epstein and Segal (1992) proposed the ex ante egalitarianism (EAE) approach. 
This approach evaluates the SWF using a concave function $\phi$, which transforms the individual expected utilities: ${ }^{22}$

$$
W^{E A E}(C)=\sum_{n \in N} q_{n} \phi\left(\sum_{s \in S} q_{s}\left[v\left(c_{n s}\right)\right]\right) .
$$

We can then show that the EAE welfare criterion (22) is ordinally equivalent to our formulation $W^{n s}(C)$ in (19), in which the inequality aversion is stronger than risk aversion (i.e. $\phi \equiv g \circ v^{-1}$ is concave). Another option, known as the ex post egalitarianism (EPE, Adler and Sanchirico, 2006), involves applying the concave function on an individual's utilities and evaluating welfare as the expected value of such transformations as

$$
W^{E P E}(C)=\sum_{s \in S} q_{s} \sum_{n \in N} q_{n} \phi\left[v\left(c_{n s}\right)\right] .
$$

In the context of our framework, such transformation is equivalent to considering a version of the utilitarian SWF, using a more concave function for both inequity aversion over states and individuals. Finally, a third approach, proposed by Fleurbaey (2010) and known as the expected equally distributed equivalent (EEDE) approach, computes the expected value of a SWF taking the form of an EDE(Atkinson, 1970):

$$
\left.W^{E E D E}(C)=\sum_{\mathrm{s} \in \mathrm{S}} q_{s}\left[\phi^{-1}\left(\sum_{n \in N} q_{n} \phi\left[v\left(c_{s n}\right)\right]\right)\right]\right) .
$$

Note that $W^{E E D E}(C)$ is ordinally equivalent to our double equity equivalent $W^{s n}(C)$ computed in expression (20), provided that inequality aversion is stronger than risk aversion (i.e. $\phi=g \circ v^{-1}$ is concave). Finally, the EAE and EEDE criteria both collapse to the standard utilitarian criterion when inequality aversion and risk aversion coincide.

The two orders of aggregation $W^{s n}(C)$ and $W^{n s}(C)$-or, equivalently, $W^{E A E}(C)$ and $W^{E E D E}(C)$ - can also characterize situations of "global" and "individual" risk aversion, respectively. In general, global risk aversion considers the risk at the global level and therefore takes into account risk sharing possibilities permitted by the level of inequality between individuals. By contrast, individual risk aversion considers the risk at the individual level only and evaluates inequalities in terms of CEs. Therefore the order of aggregation of the SWF implicitly determines how risk is being shared

\footnotetext{
${ }^{22}$ Note that in the original version of the EAE $q_{n}$ is equal to unity (which is equivalent to $q_{n}=1 /|N|$ in our equity equivalent notation) because the individual weights are typically assumed to be uniform.
} 
by the social planner. To illustrate the difference between the two concepts, consider the simple scenario in Table 1.

\begin{tabular}{|c|c|c||c|}
\hline & state 1 & state 2 & $\begin{array}{c}\text { Expected } \\
\text { consumption }\end{array}$ \\
\hline country 1 & 10 & 20 & 15 \\
\hline country 2 & 20 & 10 & 15 \\
\hline \hline Mean & 15 & 15 & 15 \\
\hline
\end{tabular}

(a) situation $\mathrm{A}$

\begin{tabular}{|c|c|c||c|}
\hline & state 1 & state 2 & $\begin{array}{c}\text { Expected } \\
\text { consumption }\end{array}$ \\
\hline country 1 & 15 & 5 & 10 \\
\hline country 2 & 30 & 10 & 20 \\
\hline \hline Mean & 22.5 & 7.5 & 15 \\
\hline
\end{tabular}

(b) situation B

Table 1: Examples of distributions of consumption over individuals and states of the world

Imagine two individuals (in this case, represented by two countries of equal population size) and two states of the world, each one realizing with probability 0.5. Table 1 presents the consumption levels associated with these distributions, as well as the average consumption for each state, the expected consumption for each country, and the mean expected consumption $\mathbb{E}^{n} \mathbb{E}^{s}(C)$ (equal to 15 in both situations). In situation $\mathrm{A}$, the mean consumption is certain because of the perfectly negative correlation between the distributions of consumption over states. In this case, risk aversion does not play any role if the social planner considers the risk affecting global consumption only. In other words, SWF $W^{s n}(C)$-or equivalently, $W^{E E D E}(C)$ - does not depend on risk preferences. By contrast, if risk is considered for each country separately, the resulting CEs are lower than the expected level of consumption in each country, and the aggregation over both countries results in a lower level of equity equivalent consumption. Intuitively, the two concepts of EAE and EEDE yield different results when there is room for welfare enhancing, risk sharing transfers. In situation $\mathrm{B}$, the risk proportionally affects one relatively poor (country 1 ) and one relatively rich country (country 2 ). In relative terms, the degree of inequality is thus constant across states of the world so that, ex-post, country 2 always consumes twice as much as country 1.

In Table 2, we compute social welfare in terms of equity equivalents, based on isoelastic utility functions. In particular, we use relative risk $(\rho)$ and inequality $(\gamma)$ aversion coefficients that can take the value of either 0.7 or 2 . In line with Proposition 1 and its corollary, we can directly check that the order of aggregation does not matter when $\rho=\gamma$. In situation A, as we previously noted, risk aversion does not affect the welfare measures if the aggregation is first realized according to the individual dimension. In other words, $W^{s n}(C)$ and $I^{s n}$ do no depend on $\rho$. In line with Proposition 3, the order of aggregation also affects the evaluation of 


\begin{tabular}{|c|c|c|c|c|}
\hline$\gamma \rho$ & \multicolumn{2}{|c|}{0.7} & \multicolumn{2}{c|}{2} \\
\hline & $W^{s n}(C)$ & $W^{n s}(C)$ & $W^{s n}(C)$ & $W^{n s}(C)$ \\
\hline 0.7 & 14.4 & 14.4 & $\mathbf{1 4 . 4}$ & $\mathbf{1 3 . 3}$ \\
\hline 2 & $\mathbf{1 3 . 3}$ & $\mathbf{1 4 . 4}$ & 13.3 & 13.3 \\
\hline
\end{tabular}

(a) situation $\mathrm{A}$

\begin{tabular}{|c|c|c|c|c|}
\hline$\gamma$ & \multicolumn{2}{|c|}{0.7} & \multicolumn{2}{c|}{2} \\
\hline & $W^{s n}(C)$ & $W^{n s}(C)$ & $W^{s n}(C)$ & $W^{n s}(C)$ \\
\hline 0.7 & 13.0 & 13.0 & $\mathbf{1 2 . 1}$ & $\mathbf{1 2 . 1}$ \\
\hline 2 & $\mathbf{1 0 . 8}$ & $\mathbf{1 0 . 8}$ & 10.0 & 10.0 \\
\hline
\end{tabular}

(b) situation B

Table 2: Welfare and inequity measures with different orders of aggregation and relative inequity aversion coefficients

social welfare. In situation A, for example, $W^{s n}(C)>W^{n s}(C)$ when $\rho>\gamma$. We can then easily compute the levels of inequity for each order of aggregation using formula (10) as relative differences from the expected value (which equals 15 in both situations). In situation B, regardless of whether risk is considered at the global or the individual level, the same level of social welfare, $W^{s n}(C)=W^{n s}(C)$ (and thus of inequity), emerges. The reason for this is that the consumption stream is multiplicative over countries, as in part (iii) of Proposition 3.

Extension to time and individual While we followed two strands of literatures when making explicit the distinction between prioritarianism criteria (when different generations are considered -in our notation the time dimension) and egalitarianism criteria (when different individuals are considered), note that the distinction may seem artificial from a welfare perspective. Indeed, the welfare economic literature does not usually distinguish between individuals living contemporaneously and individuals living at different periods of time. Yet, in some cases, the distinction between the individual and time dimensions may be warranted. For example, such distinction enables separately considering the issues of intertemporal inequity (among generations of individuals) and inequality (among individuals living at the same period).

Under the general framework we propose, two orders of aggregations emerge as measures of welfare across individuals and time. Following previous notations, we can write these as $W^{t n}(C)$ and $W^{n t}(C)$ or more specifically as

$$
W^{t n}(C)=u^{-1}\left(\sum_{\mathrm{t} \in \mathrm{T}} q_{t} u \circ g^{-1}\left(\sum_{n \in N} q_{n} g\left(c_{t n}\right)\right)\right)
$$

and

$$
W^{n t}(C)=g^{-1}\left(\sum_{n \in N} q_{n} g \circ u^{-1}\left(\sum_{t \in T} q_{t} u\left(c_{n t}\right)\right)\right) .
$$

The distinction between the individual and time dimensions from a normative standpoint is not trivial. Yet the literature focusing on inequality measurement has ex- 
plicitly examined the possibility of different functions capturing attitudes toward the individual and time dimensions (e.g., Atkinson and Bourguignon, 1982). Moreover, some arguments may be advanced to justify such a distinct treatment of the two dimensions. Imagine the case in which a distinction is made between different countries (individual dimension), each consisting of a dynasty made up of different generations (time dimension). In a situation in which socio-economic decisions are taken at the national level, such a situation would result in decisions affecting the time but not the individual dimension. How should the welfare be evaluated in this case? If attitudes toward the two dimensions are identical, the order of aggregation is irrelevant, and the welfare measure (discounted utilitarian) is essentially equivalent to the one adopted in many (deterministic) empirical models examining regionally disaggregated welfare problems. This is the case in most of the so-called integrated assessment models of climate change, which are based on welfare maximization and analyze the interaction between climate change and the economy. Yet, although it may seem relevant to treat the inequity attitude across time and state distinctively, expressions (25) and (26) reveal different interpretations. For example, in $W^{t n}(C)$, where the aggregation is first realized across countries and then over time, we can show that under inequality neutrality, the inner part of the welfare criterion simply becomes the average global per-country consumption level at time $t$. Under this assumption, the welfare criterion is equivalent to the idea proposed by Stanton (2011) of simply maximizing the sum of consumption across countries. The other possible order of aggregation, $W^{n t}(C)$, is a weighted sum, taken today, over the utility of dynasties in each country.

\section{Extension to three dimensions}

Given the previous discussion on the separation of the individual and time dimensions, a natural extension allowed by our general framework is to take welfare as equity equivalents along the three dimensions of individual, time, and state. In this case, the SWF would directly follow from extending the aforementioned twodimensional analysis.

Definition 6. The SWF over the ordered dimensions $i, j$, and $k$ is the function $W^{i j k}: \mathcal{C} \rightarrow \mathbb{R}^{+}$defined as

$$
W^{i j k}(C) \equiv \mathcal{E}^{i}\left(\mathcal{E}^{j}\left(\mathcal{E}^{k}(C)\right)\right) \quad \forall i, j, k \in\{s, t, n\} \text { with } i \neq j \neq k .
$$


As indicated by the order of superscripts, this SWF would represent the preferences of a social planner who first aggregates consumption through dimension $k$, then through dimension $j$, and finally through dimension $i$. As before, the standard WU equivalent consumption represents the preferences of a social planner whose attitudes toward the three dimensions are identical. This relatively tractable welfare function is ordinally equivalent to that typically used in applied economic models. In this case, the order of aggregation does not alter the social welfare evaluation. Yet, as should be clear from criterion (27) and the discussion in the two-dimensional case, the order of aggregation over the different dimensions is relevant from the social planner's perspective. In particular, given different attitudes toward the different dimensions, $3 !=6$ potential different welfare orders of aggregation are admissible. ${ }^{23}$ In Appendix D, we consider some of these special cases in more details.

\section{Recursive representations and the special treat- ment of time}

While we have, thus far, treated the three dimensions analogously, we now pay particular attention to the time dimension. The time dimension presents a "natural" order over its elements and is possibly unbounded (e.g. when infinitely-lived agents are considered). These special features give rise to a recursive representation of social preferences in this dimension. In what follows, we relax the nested additive separability structure of our equity equivalent in the time dimension and allow for a recursive, non-additive representation of social preferences. This representation is particularly useful when time and state are jointly considered in dynamic setups; this alternative representation also allows us to extend our analysis to incorporate general representations that have been widely used in the literature (e.g. preferences with more than two periods; see Kreps and Porteus, 1978; Epstein and Zin, 1989; Weil, 1989, 1990).

\footnotetext{
${ }^{23}$ One of these orders can be linked to an extended BGE referred to as the "certainty, equity, and balanced growth equivalent" (CEBGE). Anthoff and Tol (2009) and Schmidt et al. (2012) use this concept in the context of climate change. It measures the level of today's consumption that, provided it were certain, equally distributed, and grew at a constant growth rate $\lambda$, would yield the same level of WU welfare as the actual consumption path (subject to inequality and uncertainty). In particular, our welfare measure in this case can be computed as $W^{i j k}(C)=\mathcal{E}^{t}\left(C E B G E(1+\lambda)^{t}\right)$. If $f_{t}$ is isoelastic and $\lambda=0$, then $C E B G E=W^{i j k}(C)$. In this sense, the three-dimensional welfare function of (8) can be considered a special case of the CEBGE with a zero growth rate.
} 
Recursive representation in the one dimensional case The one-dimensional criterion (3) we propose is defined over all the elements of the set to which the superscript $i$ refers. Yet, in some instances, it might be useful to characterize welfare recursively in the time dimension. This can be done by letting $\hat{W}_{t}^{t}(C)$ be the recursive form (as indicated by the hat) of the SWF over dimension $t$ (as denoted by the superscript) evaluated at date $t$ (as denoted by the running index $t$ as subscript). We defined this as

$$
\hat{W}_{t}^{t}(C)=\mathcal{F}\left(c_{t}, \hat{W}_{t+1}^{t}\right),
$$

where $\mathcal{F}: \mathbb{R}^{2} \rightarrow \mathbb{R}^{+}$is a so-called time aggregator in the sense of Koopmans (1960). It takes the form

$$
\mathcal{F}(x, y)=u^{-1}\left(u(x)+\widehat{q}_{t+1} u(y)\right),
$$

where $\hat{q}_{t+1}$ is the weight attached to element $t+1$ relative to element $t$ (typically constant and equal to $\beta$ in the exponential discounting model). When we evaluate SWF starting from the first period, we can show that expressions (3) and (28) are equivalent.

Recursive representation in the multi-dimensional case For general problems involving the time and state dimensions, the equity equivalent representation (8) we propose may however be dynamically inconsistent. Specifically, if risk is present in different periods, our formulation $W^{t s}(C)$ in (11) implicitly assumes that the expectation over states of the world is taken in each period separately, while formulation $W^{\text {st }}(C)$ in (12) implicitly assumes the existence of single one-for-all intertemporal risk. ${ }^{24}$ Instead, a desirable property of the welfare function should be to assume that welfare at time $t$ is evaluated on the basis of the expectation of future consumption at time $t$. By allowing an agent to care intrinsically about the timing of the resolution of uncertainty, the recursive model proposed by Kreps and Porteus (1978) achieves this flexibility for the case in which the aggregation is first realized over states and then over time. Their model does not perfectly match formulation (8), but remains tractable due to recursivity. Specifically, preferences in time $t$ are built up from preferences in time $t+1$, which do not themselves depend on unrealized contingencies. In other words, the recursive formulation of the welfare function has both the desirable properties of being dynamically consistent and independent

\footnotetext{
${ }^{24}$ See Epstein and Zin (1989, pp. 950-952) for a critical analysis of Kihlstrom and Mirman's (1974) EU approach in an intertemporal setting.
} 
of both unrealized alternatives and past consumption levels. Thus, we reformulate the recursive version of the $\operatorname{SWF} W_{t}^{t s}(C)$ at time $t$ (denoted by the subscript $t$ ) as

$$
\hat{W}_{t}^{t s}(C)=\mathcal{F}\left(c_{t}, \mathcal{E}_{t}^{s}\left(\hat{W}_{t+1}^{t s}\right)\right),
$$

where $\mathcal{F}$ the time aggregator defined in $(29)$ and $\mathcal{E}_{t}^{s}$ is the $\mathrm{CE}$ operator, taken at time $t$, which is written as

$$
\mathcal{E}_{t}^{s}\left(\hat{W}_{t+1}^{t s}\right)=v^{-1}\left(\mathbb{E}_{t}^{s}\left[v\left(\hat{W}_{t+1}^{t s}\right)\right]\right) .
$$

Here, $\mathbb{E}_{t}^{s}$ is the expectation operator conditional on all the information available at time $t$. As before, we obtain the standard case of exponential discounting by setting $\hat{q}_{t+1}=\beta$ in (29), where $\beta$ is the discount factor. A standard specification of the temporal aggregator is, for example, the constant elasticity of substitution (CES) aggregator obtained when $u$ is isoelastic: $u(z)=z^{1-\eta} / 1-\eta$. Unsurprisingly, the recursive welfare formulation (30) simplifies

$$
W_{t}^{\mathrm{WU}}(C)=u^{-1}\left(\mathbb{E}_{t}^{s}\left[\sum_{\tau} \beta^{\tau} u\left(c_{t+\tau}\right)\right]\right),
$$

when $u=v$ and exponential discounting is considered. With $W^{t s}(C)$ in (11), formulation $\hat{W}_{t}^{t s}(C)$ in (30) allows for a separation of preferences between attitudes toward time and state. Marinacci and Montrucchio (2010) show that the solution of (30) is unique and globally attractive (i.e., it allows for a solution to be found iteratively starting from any initial point) when using the CES aggregator, if $v$ exhibits increasing relative risk aversion (IRRA). This includes the widely used isoelastic (or CRRA) specification, giving rise to the popular version of Epstein and Zin (1989) and Weil $(1989,1990)$. Similarly, if three or more dimensions are considered jointly, a proper dynamic setting disentangling attitudes toward different dimensions may be required. In Appendix D, we show how recursive (time consistent) formulations of the SWF in the three dimensions can be obtained when the order of aggregation through the state dimension takes place before time.

\section{Conclusion}

The concepts of economic welfare and inequity are closely intertwined. In this paper, we show that inequity encompasses different notions depending on whether consumption is spread across individuals, time, or states of the world. We analyze 
welfare and inequity in these dimensions using a generalized framework extending the WU paradigm. In particular, we propose an "equity equivalence" in each dimension as a useful measure of welfare and derive an inequity index from its value. This generalized framework enables us to gather different concepts that have been investigated separately in different dimensions in various strands of literature. We then extend the analysis to cases in which two or three dimensions are jointly considered. In these cases, the order of aggregation matters for welfare evaluation, unless the inequity preferences are identical across all dimensions. In particular, when inequality, risk, and time-dependent distributions are jointly considered, six different orders of aggregation are possible, leading to different welfare concepts. In this case, the role of correlations across the different dimension and the characteristics of the functions representing preferences become important to rank the levels of welfare. 


\section{Appendix}

\section{A A simplex representation of the literature on the three dimensions of inequity preferences}

To complete the extensive literature review of Section 2, we provide a graphical representation of how the existing studies on inequity relate to the three dimensions and show their possible interactions in the three-dimensional simplex presented in Figure A.1.

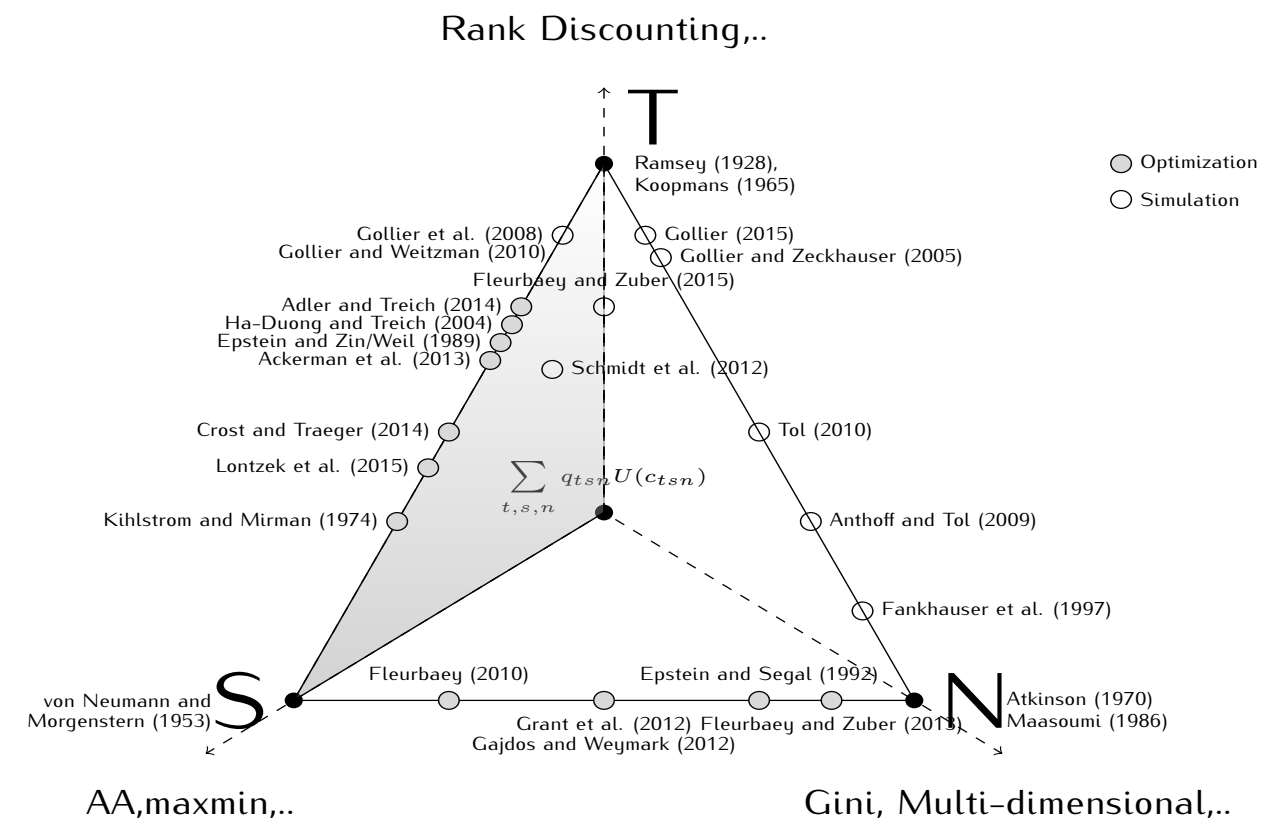

Figure A.1: A simplex representation of the extant literature on welfare across the three dimensions

In general, the location on the simplex reflects which dimension is considered and, if multiple dimensions are taken into account, how they are separated. This representation allows a broad mapping of the extant literature in the field across the three axes: at the center of the simplex, the threefold simple (weighted) sum of utilities considers all three dimensions jointly with an identical utility function across all dimensions; in other words the center represents the standard case of the WU criterion, where all dimensions are treated equally. At the three vertices, only one 
dimension is considered in isolation: for example, for the time dimension, the standard Ramsey model evaluates welfare only through time, while EU only considers utility across states, and Atkinson's measure deals with inequality issues. The three triangular areas going through the center represent two-dimensional considerations, in which welfare evaluation is, to some extent, disentangled between the two dimensions considered. The closeness to either of the three Cartesian axes represents the importance of each dimension. For example, several studies on the extended Ramsey rule include (and disentangle) both risk and time, but specifically focus on the time dimension of welfare -and thus are closer to the time axis. Finally, on the area spanning the three outward vertices, all three dimensions are considered in a disentangled fashion, as proposed in this paper, possibly with a focus on either dimension. Recall that we restrict the analysis to approaches of the utilitarian type, in which welfare can be expressed, in each dimension, as a (weighted) sum of utilities. Other approaches using more complex welfare functions in each of the dimension are indicated but go beyond the scope of this paper. We thus deliberately restrict our analysis to the boundaries of the utilitarian simplex outlined in Figure A.1.

\section{B Proofs}

Proof of Lemma 1. The proof for the case in which $f_{i}$ is strictly convex is available in Hardy et al. (1952, Theorem 106, p. 88). The case in which $f_{i}$ is strictly concave is then trivially obtained by introducing the transformation $f_{i}(x)=$ $-F_{i}(-x)$, where $F_{i}$ is increasing and convex (Hennessy and Lapan, 2006).

Proof of Proposition 2. First note that function $\phi \equiv f_{i} \circ f_{j}^{-1}$ is strictly increasing and strictly concave if and only if $-\frac{f_{i}^{\prime \prime}}{f_{i}^{\prime}}>-\frac{f_{j}^{\prime \prime}}{f_{j}^{\prime}}$. Then, we can write the condition $W^{i j}(C) \geq W^{j i}(C)$ as

$$
f_{j} \circ f_{i}^{-1}\left(\sum_{i} q_{i} f_{i} \circ f_{j}^{-1}\left(\sum_{j} q_{j} f_{j}\left(c_{i j}\right)\right)\right) \geq \sum_{j} q_{j} f_{j} \circ f_{i}^{-1}\left(\sum_{i} q_{i} f_{i}\left(c_{i j}\right)\right),
$$

which can be rewritten as

$$
\phi^{-1}\left(\sum_{i} q_{i} \phi\left(\sum_{j} q_{j} f_{j}\left(c_{i j}\right)\right)\right) \geq \sum_{j} q_{j} \phi^{-1}\left(\sum_{i} q_{i} \phi\left(f_{j}\left(c_{i j}\right)\right)\right) .
$$

From Lemma 1, we know that the equity equivalent is a concave operator so that $\mathcal{E}^{\phi}\left(\sum_{j} q_{j} f_{j}\left(c_{i j}\right)\right) \geq \sum_{j} q_{j} \mathcal{E}^{\phi}\left(f_{j}\left(c_{i j}\right)\right)$, where $\mathcal{E}^{\phi}(X) \equiv \phi^{-1} \mathbb{E}^{i} \phi(x)$. This is exactly 
what is represented in expression (B.2).

Proof of Proposition 3. When both $f_{i}$ and $f_{j}$ are of the isoelastic type with coefficients of the relative aversion $\varphi^{i}$ and $\varphi^{j}$, respectively, we can write $\phi$ as

$$
\phi(x)= \begin{cases}\frac{1}{1-\varphi^{i}}\left[\left(1-\varphi^{j}\right) x\right]^{\frac{1-\varphi^{i}}{1-\varphi^{j}}}, & \text { if } \varphi^{j} \neq 1 \\ \frac{e^{\left(1-\varphi^{i}\right) x}}{1-\varphi^{i}}, & \text { if } \varphi^{j}=1 .\end{cases}
$$

In this case, $\phi$ is itself of the harmonic absolute inequity aversion (HARA) class. In particular, it is an isoelastic function when $\varphi^{j} \neq 1$, while it is an exponential function when $\varphi^{j}=1$. In accordance with the discussion on the general HARA class provided in Appendix $\mathrm{C}$, this function is only defined on the domain $\mathbb{R}^{+}$when $\varphi^{j}<1$ and in the domain $\mathbb{R}^{-}$when $\varphi_{j}>1$. Moreover, this function $\phi$ is increasing, strictly concave when $\varphi^{i}>\varphi^{j}$, strictly convex when $\varphi^{i}<\varphi^{j}$, and linear when $\varphi^{i}=\varphi^{j}$. The results therefore directly follow from Proposition 5 and Corollary 2 presented in Appendix C. In particular, cases (i) and (iii) follow as special cases when $\tau=0$, which leads to $b=0$ (because $\phi$ is of the isoelastic type), and cases (ii) and (iv) follow as special cases when $\tau \rightarrow \infty$, which leads to $a=1$ (because $\phi$ is of the exponential type). ${ }^{25}$

Proof of Proposition 4. To simplify notations, we first write $W^{j i}(C)$ using a continuous distribution of $C$ as $W^{j i}(C)=f_{j}^{-1} \int f_{j} \circ f_{i}^{-1}\left(\int f_{i}(C) d G_{i \mid j}\right) d G_{j}$, where $d G_{i \mid j}$ denotes the conditional cumulative distribution of $C$ across dimension $i$. Given the independence assumption, $d G_{i \mid j}=d G_{i}$, such that we obtain $W^{j i}(C)=f_{j}^{-1}\left(\int f_{j} d G_{j}\right) f_{i}^{-1}\left(\int f_{i}(c) d G_{i}\right)=W^{i j}(C)$. That is, in the case of independence it holds that $W^{i j}(C)=\mathcal{E}^{i}(C) \mathcal{E}^{j}(C)=\mathcal{E}^{j}(C) \mathcal{E}^{i}(C)=W^{j i}(C)$.

Proof of Corollary 1. Applying the result of Proposition 4 to the definition in the two-dimensional inequity index presented in equation (10) gives $I^{i j}=1-\frac{\mathcal{E}^{i}(C) \mathcal{E}^{j}(C)}{\mathbb{E}^{i} \mathbb{E}^{j}[C]}$. Then, applying the definition in equation (5) simply leads to $I^{i j}=1-\left(1-I^{i}\right)(1-$ $\left.I^{j}\right)$.

\footnotetext{
${ }^{25}$ We can compute the absolute inequity tolerance of $\phi \equiv f_{j} \circ f_{i}^{-1}$ with isoelastic specifications as $\phi^{\prime}(x) /-\phi^{\prime \prime}(x)=\left(\varphi_{j}-\varphi_{i}\right)\left(1-\varphi_{i}\right) x$, which is linear in $x$.
} 


\section{Welfare comparisons in the two-dimensional HARA cases}

Most of the parametric functions used to represent preferences in economics, finance, and decision theory belong to the class of harmonic absolute inequity aversion functions. This class is best known as HARA because the dimension considered is generally the risk one. A function $f_{i}$ is of the HARA class if its absolute inequity tolerance (i.e. the inverse of its absolute inequity aversion) is linear in consumption. In particular, $f_{i}$ is of the HARA class if it can be written as:

$$
f_{i}(x)=\frac{1-\psi}{\psi}\left[\frac{\alpha x}{1-\psi}+\eta\right]^{\psi}
$$

which is defined for values of $x$, such that $\frac{\alpha x}{1-\psi}+\eta>0$, and is strictly increasing and concave when $\alpha>0$. The function is also of the isoelastic type when $\eta=0$ and of the exponential type when $\psi \rightarrow-\infty$. In the special case of HARA functions, Proposition 2 can be expressed as follows:

Proposition 5. Let $f_{i}$ and $f_{j}$ be strictly increasing, strictly concave, four times continuously differentiable functions such that $f_{i} \neq a f_{j}+b$, where $a \neq 0, b \in \mathbb{R}$, and let $\phi \equiv f_{i} \circ f_{j}^{-1}$ be of the HARA form. Then, $W^{i j}(C)=W^{j i}(C)$ if and only if

$$
y_{i j^{\prime}}=a y_{i j}+b \forall i, j, j^{\prime}
$$

where $y_{i j} \equiv f_{j}\left(c_{i j}\right), a=\left[\alpha \mathcal{E}^{\phi}\left(y_{i j^{\prime}}\right)+\tau\right] /\left[\alpha \mathcal{E}^{\phi}\left(y_{i j}\right)+\tau\right]$, $b=\left[\mathcal{E}^{\phi}\left(y_{i j^{\prime}}\right)-\mathcal{E}^{\phi}\left(y_{i j}\right)\right] \tau /\left[\alpha \mathcal{E}^{\phi}\left(y_{i j}\right)+\tau\right], \tau=(1-\psi) \eta$ and $\mathcal{E}^{\phi}(X) \equiv \phi^{-1}\left(\mathbb{E}^{i} \phi(X)\right)$.

Proof. First, $W^{i j}(C)=W^{j i}(C) \Longleftrightarrow \mathcal{E}^{\phi}\left(\sum_{j} q_{j} y_{i j}\right)=\sum_{j} q_{j} \mathcal{E}^{\phi}\left(y_{i j}\right)$. The proof for the case when there are only two elements in the set indexed by $j$ is available in Hennessy and Lapan (2006, Proposition 1 (iii), p. 3). It is then easy to extend the proof to the general cases by proceeding as follows: Subdivide $y_{i j}$ into $y_{i j}=$ $v x_{i j}+(1-v) x_{i j^{\prime}}$, with $v \in(0,1)$, and note that with $x_{i j}$ and $x_{i j^{\prime}}, W^{i j}(C)=W^{j i}(C)$ if and only if $x_{i j^{\prime}}=a^{x} x_{i j}+b^{x}$, where $a^{x}=\left[\alpha \mathcal{E}^{\phi}\left(x_{i j^{\prime}}\right)+\tau\right] /\left[\alpha \mathcal{E}^{\phi}\left(x_{i j}\right)+\tau\right]$ and $b^{x}=\left[\mathcal{E}^{\phi}\left(x_{i j^{\prime}}\right)-\mathcal{E}^{\phi}\left(x_{i j}\right)\right] \tau /\left[\alpha \mathcal{E}^{\phi}\left(x_{i j}\right)+\tau\right]$. When considering $y_{i j}$ and $y_{i j^{\prime}}$, we therefore have $y_{i j^{\prime}}=a\left(v x_{i j}+(1-v) x_{i j^{\prime}}\right)+b=a\left(v x_{i j}+(1-v)\left(a^{x} x_{i j}+b^{x}\right)\right)+b$. Algebra enables us to write this as $y_{i j^{\prime}}=a^{y} x_{i j}+b^{y}$, where $a^{y}=\left[\alpha \mathcal{E}^{\phi}\left(y_{i j^{\prime}}\right)+\tau\right] /\left[\alpha \mathcal{E}^{\phi}\left(x_{i j}\right)+\tau\right]$ and $b^{y}=\left[\mathcal{E}^{\phi}\left(y_{i j^{\prime}}\right)-\mathcal{E}^{\phi}\left(x_{i j}\right)\right] \tau /\left[\alpha \mathcal{E}^{\phi}\left(x_{i j}\right)+\tau\right]$. Thus, if we let $x_{i j} \equiv y_{i j^{\prime \prime}}$ and $x_{i j^{\prime}} \equiv y_{i j^{\prime \prime \prime}}$, the Proposition's statement holds when there are three elements in the set indexed by $j$. Repeating this procedure gives the result. 
The combination of Propositions 2 and 5 leads us to the following corollary:

Corollary 2. Let $f_{i}$ and $f_{j}$ be strictly increasing, strictly concave, four times continuously differentiable functions such that $f_{i} \neq a f_{j}+b$, where $a \neq 0, b \in \mathbb{R}$, and let $\phi \equiv f_{i} \circ f_{j}^{-1}$ be of the HARA form and the conditions of Proposition 5 not to hold, then $W^{i j}(C)>W^{j i}(C)$ if and only if $f_{i}$ is strictly more concave than $f_{j}$.

Proof. If $\phi$ is of the HARA form, its absolute risk tolerance is linear in consumption, and we therefore know from Proposition 2 case (i) that $W^{i j}(C) \geq W^{j i}(C)$ if and only if $f_{i}$ is strictly more (less) concave than $f_{j}$. Moreover, from Proposition 5 and the properties of HARA functions, we also know that $W^{i j}(C)=W^{j i}(C)$ only holds under specific conditions when $f_{i} \neq a f_{j}+b$, where $a \neq 0, b \in \mathbb{R}$. Combining these two results therefore proves Corollary 2.

\section{Welfare orderings in three dimensions}

As outlined in the main body of the paper, six welfare orderings are admissible in the three-dimensional case. Table D.1 summarizes these six orderings of $W^{i j k}(C)$ in terms of how the dimensions are aggregated.

\begin{tabular}{|c|c|c|c|c|c|}
\hline SWF & Outer & Intermediate & Inner & $\begin{array}{c}\text { Description } \\
\text { interpretation }\end{array}$ & $\begin{array}{c}\text { Time consistent } \\
\text { recursive form }\end{array}$ \\
\hline \hline$W^{t s n}(C)$ & $\mathrm{T}$ & $\mathrm{S}$ & $\mathrm{N}$ & "global risk aversion" & $\mathrm{Y}$ \\
\hline$W^{t n s}(C)$ & $\mathrm{T}$ & $\mathrm{N}$ & $\mathrm{S}$ & "individual risk aversion" & $\mathrm{Y}$ \\
\hline$W^{\text {nts }}(C)$ & $\mathrm{N}$ & $\mathrm{T}$ & $\mathrm{S}$ & dynastic recursive preferences & $\mathrm{Y}$ \\
\hline$W^{\text {nst }}(C)$ & $\mathrm{N}$ & $\mathrm{S}$ & $\mathrm{T}$ & dynastic welfare of KM ${ }^{26}$ preferences & $\mathrm{N}$ \\
\hline$W^{\text {stn }}(C)$ & $\mathrm{S}$ & $\mathrm{T}$ & $\mathrm{N}$ & KM preferences, intertemporal welfare & $\mathrm{N}$ \\
\hline$W^{\text {snt }}(C)$ & $\mathrm{S}$ & $\mathrm{N}$ & $\mathrm{T}$ & KM preferences over dynastic welfare & $\mathrm{N}$ \\
\hline
\end{tabular}

Table D.1: Possible orders of aggregation of the SWF in three dimensions

As the table shows, an ordering in which the aggregation over states of the world is taken after the time aggregation does not admit a recursive time-consistent form. In what follows, we consider the first three orderings in more details.

Global risk aversion We first consider the case $W^{t s n}(C)$. It could be interpreted as an instance in which the social planner considers welfare from a global perspective, when evaluating different states of the world. This social planner is referred to being

\footnotetext{
${ }^{26} \mathrm{KM}$ refers to Kihlstrom and Mirman (1974) preferences when expectation is taken over the full intertemporal stream of consumption.
} 
"globally risk averse" in the sense that she considers risky payoffs at the aggregated level (i.e. over several individuals). Yet the difference in the global risk aversion problem discussed in Section 4.3 is that a time dimension is also considered. (An alternative way to show the same problem is to start from the the Kreps-Porteus formulation $\hat{W}_{t}^{t s}(C)$ for different individuals, so that the $\operatorname{EDE} \mathcal{E}^{n}(C)$ is itself risky.) In this case, we can write the time-consistent version of welfare function $W_{t}^{t s n}(C)$ recursively as

$$
\hat{W}_{t}^{t s n}(C)=\mathcal{F}\left(\mathcal{E}^{n}\left(C_{t}\right), \mathcal{E}_{t}^{s}\left(\hat{W}_{t+1}^{t s n}\right)\right),
$$

where, as before, $\mathcal{F}$ is the temporal aggregator defined as in (29) and $\mathcal{E}_{t}^{s}$ is the time $t$ CE operator. ${ }^{27}$ Intuitively, global risk aversion assumes that the social planner has access to risk sharing technology (within the bounds of her inequality attitude). In particular, this means that negatively correlated risks can be mediated (see also the application in Emmerling, 2018).

Individual risk aversion The second order of aggregation we consider is $W^{\text {tns }}(C)$. Compared with the previous case, the orders of aggregation across individuals and states of the world are inverted here. This means that the social planner, instead, considers individual risky consumption and then aggregates the individual CEs across individuals and time. In this case, the social planner exhibits individual risk aversion. In such a situation, the recursive form of the welfare function takes the form

$$
\hat{W}_{t}^{t n s}(C)=\mathcal{F}^{t}\left(c_{t}, \mathcal{E}^{n}\left(\mathcal{E}_{t}^{s}\left(\hat{W}_{t+1}^{t n s}\right)\right)\right)
$$

The implication of having such an SWF is that if risk aversion increases, the implicit welfare weight increases for the individual having the lowest level of consumption in any state of the world.

Dynastic recursive preferences Finally, if the aggregation over countries is realized after the intertemporal aggregation over time, global inequality is evaluated by considering, for each individual, the welfare computed over an intertemporal time horizon. In this case, we can write the time consistent version of the recursive welfare function as

\footnotetext{
${ }^{27}$ In the special case in which the social planner is inequality neutral, it is nothing but the adjusted average consumption taken over the different individuals. In this case, the $\operatorname{SWF} \hat{W}_{t}^{\text {tsn }}(C)$ becomes a version of (30) computed for the average per-individual consumption.
} 


$$
\hat{W}_{t}^{n t s}(C)=\mathcal{E}^{n}\left(\left(\hat{W}_{t}^{t s}(C)\right)_{n \in N}\right)
$$

where $\left(\hat{W}_{t}^{t s}(C)\right)_{n \in N}$ is the Kreps-Porteus recursive SWF defined in expression (30), but is defined for each individual separately. That is, the social planner aggregates the welfare of the different individuals, all of whom consider weighted utility over their lifetime. 


\section{References}

Ackerman, F., E. A. Stanton, and R. Bueno (2013, September). Epstein-Zin Utility in DICE: Is Risk Aversion Irrelevant to Climate Policy? Environmental and Resource Economics 56(1), 73-84.

Adler, M. (2012, December). Well-Being and Fair Distribution: Beyond Cost-Benefit Analysis. New York: Oxford University Press.

Adler, M. and N. Treich (2014). Consumption, Risk and Prioritarianism. TSE Working Paper 14-500, Toulouse School of Economics (TSE).

Adler, M. D. and M. Fleurbaey (Eds.) (2016, June). The Oxford Handbook of WellBeing and Public Policy. Oxford Handbooks. Oxford, New York: Oxford University Press.

Adler, M. D. and C. W. Sanchirico (2006). Inequality and uncertainty: Theory and legal applications. Univ.Pennsylvania Law Review 155, 279.

Adler, M. D. and N. Treich (2017). Utilitarianism, prioritarianism, and intergenerational equity: A cake eating model. Mathematical Social Sciences 87, 94-102.

Anthoff, D. and R. S. J. Tol (2009, March). The Impact of Climate Change on the Balanced Growth Equivalent: An Application of FUND. Environmental and Resource Economics 43(3), 351-367.

Aristei, D. and B. Bracalente (2011). Measuring multidimensional inequality and well-being: methods and an empirical application to Italian regions. Statistica $71(2), 239-266$.

Arrow, K. J. (1964). The role of securities in the optimal allocation of risk-bearing. Review of Economic Studies 31(2), 91-96.

Atkinson, A. B. (1970). On the measurement of inequality. Journal of Economic Theory 2(3), 244-263.

Atkinson, A. B. and F. Bourguignon (1982, April). The Comparison of MultiDimensioned Distributions of Economic Status. The Review of Economic Studies $49(2), 183-201$.

Baumol, W. J. (1968). On the Social Rate of Discount. The American Economic Review 58(4), 788-802.

Berger, L., J. Emmerling, and M. Tavoni (2017). Managing catastrophic climate risks under model uncertainty aversion. Management Science 63(3), 749-765.

Bommier, A. (2007). Risk Aversion, Intertemporal Elasticity of Substitution and Correlation Aversion. Economics Bulletin 4(29).

Bommier, A., A. Chassagnon, and F. Le Grand (2012). Comparative risk aversion: A formal approach with applications to saving behavior. Journal of Economic Theory 147(4), 1614-1641.

Bosmans, K., K. Decancq, and E. Ooghe (2015, October). What do normative indices of multidimensional inequality really measure? Journal of Public Economics 130, 94-104. 
Crost, B. and C. P. Traeger (2014, June). Optimal CO2 mitigation under damage risk valuation. Nature Climate Change 4(7), 631-636.

Dalton, H. (1920). The Measurement of the Inequality of Incomes. The Economic Journal 30(119), 348-361.

Decancq, K., A. Decoster, and E. Schokkaert (2009, January). The Evolution of World Inequality in Well-being. World Development 37(1), 11-25.

Decancq, K. and M. A. Lugo (2012). Inequality of Wellbeing: A Multidimensional Approach. Economica 79(316), 721-746.

Diamond, P. A. et al. (1967). Cardinal welfare, individualistic ethics, and interpersonal comparison of utility: Comment. The Journal of Political Economy 75(5), 765 .

Eeckhoudt, L., C. Gollier, and H. Schlesinger (2005). Economic and financial decisions under risk. Princeton University Press.

Emmerling, J. (2018, June). Sharing of climate risks across world regions. Climate Change Economics 09(03), 1-19.

Epstein, L. G. and U. Segal (1992, August). Quadratic Social Welfare Functions. Journal of Political Economy 100(4), 691-712.

Epstein, L. G. and S. E. Zin (1989, July). Substitution, Risk Aversion, and the Temporal Behavior of Consumption and Asset Returns: A Theoretical Framework. Econometrica 57(4), 937-969.

Fankhauser, S., R. S. Tol, and D. W. Pearce (1997, October). The Aggregation of Climate Change Damages: a Welfare Theoretic Approach. Environmental and Resource Economics 10(3), 249-266.

Feng, T. and S. Ke (2018). Social Discounting and Intergenerational Pareto. Econometrica 86(5), 1537-1567.

Fleming, M. (1952, August). A Cardinal Concept of Welfare. The Quarterly Journal of Economics 66(3), 366-384.

Fleurbaey, M. (2010). Assessing Risky Social Situations. Journal of Political Economy 118(4), 649-680.

Fleurbaey, M. and S. Zuber (2013, November). Inequality aversion and separability in social risk evaluation. Economic Theory 54(3), 675-692.

Fleurbaey, M. and S. Zuber (2015, August). Discounting, risk and inequality: A general approach. Journal of Public Economics 128, 34-49.

Foster, J. E., L. F. Lopez-Calva, and M. Szekely (2005, March). Measuring the Distribution of Human Development: methodology and an application to Mexico. Journal of Human Development 6(1), 5-25.

Gajdos, T. and E. Maurin (2004, May). Unequal uncertainties and uncertain inequalities: an axiomatic approach. Journal of Economic Theory 116(1), 93-118. 
Gajdos, T. and J. A. Weymark (2005, October). Multidimensional generalized Gini indices. Economic Theory 26(3), 471-496.

Gajdos, T. and J. A. Weymark (2012, July). Introduction to inequality and risk. Journal of Economic Theory 147(4), 1313-1330.

Gollier, C. (2001). The Economics of Risk and Time. The MIT Press.

Gollier, C. (2008, December). Discounting with fat-tailed economic growth. Journal of Risk and Uncertainty 37(2), 171-186.

Gollier, C. (2015, January). Discounting, inequality and economic convergence. Journal of Environmental Economics and Management 69, 53-61.

Gollier, C., P. Koundouri, and T. Pantelidis (2008). Declining Discount Rates: Economic Justifications and Implications for Long-Run Policy. Economic Policy 23(56), 757-795.

Gollier, C. and M. L. Weitzman (2010). How should the distant future be discounted when discount rates are uncertain? Economics Letters 107(3), 350-353.

Gollier, C. and R. Zeckhauser (2005). Aggregation of Heterogeneous Time Preferences. Journal of Political Economy 113(4), 878-896.

Grant, S., A. Kajii, B. Polak, and Z. Safra (2012, July). Equally-distributed equivalent utility, ex post egalitarianism and utilitarianism. Journal of Economic Theory $147(4), 1545-1571$.

Ha-Duong, M. and N. Treich (2004, June). Risk Aversion, Intergenerational Equity and Climate Change. Environmental and Resource Economics 28(2), 195-207.

Hardy, G. H., J. E. Littlewood, and G. Pólya (1952). Inequalities. Cambridge university press.

Harsanyi, J. C. (1955). Cardinal Welfare, Individualistic Ethics, and Interpersonal Comparisons of Utility. Journal of Political Economy 63(4), 309-321.

Hennessy, D. A. and H. E. Lapan (2006). On the nature of certainty equivalent functionals. Journal of Mathematical Economics 43(1), 1-10.

Kihlstrom, R. E. and L. J. Mirman (1974, July). Risk aversion with many commodities. Journal of Economic Theory 8(3), 361-388.

Klibanoff, P., M. Marinacci, and S. Mukerji (2005, November). A Smooth Model of Decision Making under Ambiguity. Econometrica 73(6), 1849-1892.

Koopmans, T. (1960). Stationary ordinal utility and impatience. Econometrica: Journal of the Econometric Society, 287-309.

Kreps, D. M. and E. L. Porteus (1978, January). Temporal Resolution of Uncertainty and Dynamic Choice Theory. Econometrica 46(1), 185-200.

Lontzek, T. S., Y. Cai, K. L. Judd, and T. M. Lenton (2015, May). Stochastic integrated assessment of climate tipping points indicates the need for strict climate policy. Nature Climate Change 5(5), 441-444. 
Maasoumi, E. (1986, July). The Measurement and Decomposition of MultiDimensional Inequality. Econometrica 54(4), 991.

Marinacci, M. (2015, December). Model Uncertainty. Journal of the European Economic Association 13(6), 1022-1100.

Marinacci, M. and L. Montrucchio (2010). Unique solutions for stochastic recursive utilities. Journal of Economic Theory 145(5), 1776-1804.

Maskin, E. (1978). A theorem on utilitarianism. The Review of Economic Studies 45(1), 93-96.

Mirrlees, J. A. and N. H. Stern (1972, April). Fairly good plans. Journal of Economic Theory 4 (2), 268-288.

Nordhaus, W. and P. Sztorc (2013). Dice 2013r: Introduction and user's manual. retrieved November.

Nordhaus, W. D. (1993). Optimal greenhouse-gas reductions and tax policy in the" dice" model. The American Economic Review, 313-317.

Nordhaus, W. D. and Z. Yang (1996, September). A Regional Dynamic GeneralEquilibrium Model of Alternative Climate-Change Strategies. The American Economic Review 86(4), 741-765.

Piacquadio, P. G. (2014, September). Intergenerational egalitarianism. Journal of Economic Theory 153, 117-127.

Pigou, A. C. (1912). Wealth and welfare. Macmillan and Company, limited.

Pratt, J. (1964). Risk Aversion in the Small and in the Large. Econometrica 32(1), 122-136.

Ramsey, F. P. (1928, December). A Mathematical Theory of Saving. The Economic Journal 38(152), 543-559.

Schmidt, M. G. W., H. Held, E. Kriegler, and A. Lorenz (2012, August). Climate Policy Under Uncertain and Heterogeneous Climate Damages. Environmental and Resource Economics 54(1), 79-99.

Selden, L. (1978). A new representation of preferences over "certain x uncertain" consumption pairs: The "ordinal certainty equivalent" hypothesis. Econometrica 46(5), 1045-1060.

Selden, L. (1979). An oce analysis of the effect of uncertainty on saving under risk preference independence. The Review of Economic Studies 46(1), 73-82.

Stanton, E. A. (2011). Negishi welfare weights in integrated assessment models: the mathematics of global inequality. Climatic Change 107(3-4), 417-432.

Tol, R. S. (2010). International inequity aversion and the social cost of carbon. Climate Change Economics (CCE) 1(01), 21-32.

Tsui, K.-Y. (1995). Multidimensional Generalizations of the Relative and Absolute Inequality Indices: The Atkinson-Kolm-Sen Approach. Journal of Economic Theory 67(1), 251-265. 
von Neumann, J. and O. Morgenstern (1944). Theory Of Games And Economic Behavior. Princeton University Press.

Weil, P. (1989). The equity premium puzzle and the risk-free rate puzzle. Journal of Monetary Economics 24(3), 401-421.

Weil, P. (1990, February). Nonexpected Utility in Macroeconomics. The Quarterly Journal of Economics 105(1), 29-42.

Weymark, J. A. (2006). The normative approach to the measurement of multidimensional inequality. Inequality and economic integration, 303-328.

Zuber, S. and G. B. Asheim (2012, July). Justifying social discounting: The rankdiscounted utilitarian approach. Journal of Economic Theory 147(4), 1572-1601. 